\title{
Driven inelastic Maxwell models with high energy tails
}

\author{
M. H. Ernst* \\ Instituut voor Theoretische Fysica, Universiteit Utrecht, Postbus 801953508 TD Utrecht, The Netherlands \\ R. Brito ${ }^{\dagger}$ \\ Departamento de Física Aplicada I, Universidad Complutense, 28040 Madrid, Spain
}

(Received 31 December 2001; published 26 March 2002)

\begin{abstract}
The solutions of the homogeneous nonlinear Boltzmann equation for inelastic Maxwell models, when driven by different types of thermostats, show, in general, overpopulated high energy tails of the form $\sim \exp (-a c)$, with power law tails and Gaussian tails as border line cases. The results are compared with those for inelastic hard spheres, and a comprehensive picture of the long time behavior in freely cooling and driven inelastic systems is presented.
\end{abstract}

DOI: 10.1103/PhysRevE.65.040301

PACS number(s): 45.70.-n, 05.20.Dd

\section{INTRODUCTION}

Velocity distributions $f(v, t)$ with overpopulated high energy tails have been observed in many particle systems with inelastic interactions, such as granular materials, and have been studied experimentally, as well as by computer simulations and analytical methods [1]. In experimental studies energy has to be supplied at a constant rate to keep the system in a nonequilibrium steady state, while in the other approaches freely cooling systems can be studied as well. Without energy input the velocity distribution will approach a Dirac delta function $\delta(\mathbf{v})$ at large time, and consequently all its moments will vanish, including the root mean square (rms) velocity $v_{o}(t)$.

In freely cooling systems an interesting structure is revealed when velocities $\mathbf{c}=\mathbf{v} / v_{o}(t)$ are measured in units of the rms velocity, and the long time limit is taken while keeping $\mathbf{c}$ constant, the so-called scaling limit. In this limit the rescaled velocity distribution of the homogeneous cooling state can be collapsed on a scaling form or similarity solution $\widetilde{f}(c)$, which exhibits overpopulated power law tails $\sim 1 / c^{2 a+d}$ as recently discovered in $d$-dimensional inelastic Maxwell models (IMM) [2-4], or stretched exponential tails, $\sim \exp \left(-a c^{b}\right)$ with $b=1$ in inelastic hard sphere (IHS) systems [5-9].

This implies that we are dealing with nonuniform approaches to limiting behavior, i.e., nonuniformities in the limits of long times, large velocities, and vanishing inelasticity. So, one may obtain different results when taking the limits in different orders, when taking coupled limits, such as the scaling limit (as manifest in the differences between bulk and tail behavior), or when performing an expansion in powers of the inelasticity first and next the large time limit is taken (typically Gaussian tails are observed), or when taking the long time limit at fixed inelasticity (typically overpopulated tails are observed) with a whole wealth of coupled limits in between, as shown by Barrat et al. [9] for the one-

\footnotetext{
*Email address: ernst@phys.uu.nl

†Email address: brito@seneca.fis.ucm.es
}

dimensional IHS system driven by Gaussian white noise, by taking the scaling limit $\alpha \uparrow 1$ while keeping $\left(1-\alpha^{2}\right)^{1 / 3} v$ fixed.

The goal of this paper is to develop a similar picture for the structure of the velocity distribution function in the nonequilibrium steady state (NESS) in driven inelastic systems, and to show the existence of overpopulated high energy tails. This will be done by analyzing the solution of the nonlinear Boltzmann equation in the NESS of driven inelastic Maxwell models, and by combining these results with the available information on driven IMM and IHS systems. To carry out this analysis we use an asymptotic method, developed by van Noije and one of the authors [6]. This method gives robust results in the IHS case, as confirmed in great detail by the numerical studies listed above.

It is well known that in inelastic hard sphere systems, driven by Gaussian white noise, the tail in the velocity distribution is a stretched exponential $\sim \exp \left(-a c^{b}\right)$ with $b$ $=3 / 2$ [6-9]. The available results on driven inelastic Maxwell models seem to be contradictory. On the one hand the exact solution for a one-dimensional model shows an exponential tail [23]. Other results, however, suggest that the tails are Gaussian $[3,10]$. The latter results have been derived [10] by analyzing the NESS distribution function after performing an expansion in powers of the inelasticity, defined as $\left(1-\alpha^{2}\right)$, where $\alpha$ is the coefficient of restitution. However, tails may change depending on the order in which the nonuniformly convergent limits are taken [9], as we will also see in the case of driven systems in this paper. Moreover, the concept overpopulation is defined by comparing the asymptotic forms of $[\ln f(v)]$ with the corresponding form of the Gaussian distribution, and not by the behavior of the kurtosis or some higher cumulants.

Unfortunately, there is little universality in tails. The presence and also the absence of overpopulated tails depend strongly on the mode of energy input or the type of thermostat used (Gaussian tails in an ideal gas heat bath [11], and stretched exponential tails when coupled to a white noise thermostat [6]), on the type of interactions, e.g., inelastic hard spheres versus inelastic Maxwell models, and on the presence and absence of spatial fluctuations. In fact, at a very low rate of energy supply one even observes a crossover from freely evolving behavior to the driven one, as shown in Ref. [12]. The generic feature is here overpopulation of high 
energy tails created by inelastic interactions in systems with initially Gaussian tails, rather than the specific shape of the tail.

However, as soon as spatial fluctuations are present in the initial state $f(v, r, 0)$ the homogeneous cooling state of IMM and IHS becomes unstable, and the dissipative systems exhibit the clustering instability [13], and the phase separates into cold dense clusters and hot dilute regions. In this context Baldassarri et al. have investigated in Ref. [2a] what happens in the presence of spatial fluctuations in a many-body granular lattice Maxwell model, where the local density remains constant by construction, and hence the clustering instability is suppressed. Here the mean field theory would also predict power law tails for the homogeneous cooling state. Barrat et al. [9] also considered the influence of spatial fluctuations in the one-dimensional many-body IHS system, either freely evolving or driven by Gaussian white noise, using molecular dynamics, and compared their results with the direct simulation Monte Carlo (DSMC) method and an exact numerical method for solving the Boltzmann equation. They found even more subtle effects of nonuniform convergence when taking the scaling limit mentioned above.

The plan of the paper is as follows. In Sec. II the nonlinear Boltzmann equation for IMM systems is described, as well as the different types of thermostats, and following an observation of Montanero and Santos [8] we map a freely cooling IMM system on the corresponding IMM, driven by a Gaussian thermostat. So freely cooling systems are just a special case of driven systems, and we can restrict the whole analysis to IMM and IHS, driven by a variety of thermostats. In Sec. III the resulting tails are shown to be either of power law type $[2-4,14]$, or of exponential type, depending on the type of thermostats used. We conclude with some conclusions and perspectives.

\section{KINETIC EQUATIONS FOR DRIVEN DISSIPATIVE SYSTEMS}

To investigate these NESS in driven systems we analyze the nonlinear Boltmann equation in the simple case of an initially homogeneous and isotropic velocity distribution $f(v, 0)$, where $f(v, t)$ remains homogeneous and isotropic. This can be done by adding a stochastic or deterministic force to the microscopic equations of motion, which manifests itself in the Boltzmann equation as a source term $\mathcal{F} f$, i.e.,

$$
\begin{aligned}
\partial_{t} f(\mathbf{v})+\mathcal{F} f(\mathbf{v}) & =I(f) \\
& =\int_{\mathbf{n}} \int d \mathbf{v}_{2} g\left(\hat{\mathbf{v}}_{12} \cdot \mathbf{n}\right)\left[\frac{1}{\alpha} f_{1}^{* *} f_{2}^{* *}-f_{1} f_{2}\right] .
\end{aligned}
$$

Here $I(f)$ is the nonlinear Boltzmann collision term, and the second equality specializes the collision term to inelastic Maxwell models, which are characterized by a collision frequency $g\left(\hat{\mathbf{v}}_{12} \cdot \mathbf{n}\right)$ independent of the kinetic energy of the colliding particles, and $\hat{\mathbf{a}}=\mathbf{a} / a$ is a unit vector. The motivation for and the construction of these models has been dis- cussed in $[15,4]$, where also the origin of the factor $1 / \alpha$ is explained. Here $f_{1}^{* *}$ is short for $f\left(\mathbf{v}_{1}^{* *}, t\right)$, and we have absorbed constant factors in the time scale. The velocities and time have been dimensionalized in terms of the rms velocity and the mean free time in the initial state, and the dimensionless collision rate $g(\mu)$ is normalized such that $\int_{\mathbf{n}} g\left(\hat{\mathbf{v}}_{12} \cdot \mathbf{n}\right)$ $=1$. Moreover, $\int_{\mathbf{n}}(\cdots)=\left(1 / \Omega_{d}\right) \int d \mathbf{n}(\cdots)$ is an average over a $d$-dimensional unit sphere, where $\Omega_{d}=2 \pi^{d / 2} / \Gamma\left(\frac{1}{2} d\right)$. In the Maxwell models discussed here we have either $g(\mu)$ $=1$ (as used in [2-4]), or $g(\mu)=C|\mu|$ (as in $[15,10,4,14]$ ).

The postcollision velocities $\mathbf{v}_{i}^{*}$ with $i, j=\{1,2\}$ are defined as

$$
\mathbf{v}_{i}^{*}=\mathbf{v}_{i}^{*}(\alpha)=\mathbf{v}_{i}-\frac{1}{2}(1+\alpha) \mathbf{v}_{i j} \cdot \mathbf{n n},
$$

and the restituting velocities, appearing in the collision term (1), are given by $\mathbf{v}_{i}^{* *}=\mathbf{v}_{i}^{*}(1 / \alpha)$. Here $\alpha$ is the coefficient of restitution $(0<\alpha<1)$, and $\mathbf{n}$ is a unit vector along the line of centers of the interacting particles. In one dimension the angular average $\int_{\mathbf{n}}$, as well as the tensorial product $\mathbf{n n}$ can be replaced by 1 . The Boltzmann collision operator conserves the number of particles and momentum, but not the energy, and normalizations are chosen such that $\langle 1\rangle=1$ and $\left\langle v^{2}\right\rangle$ $=\frac{1}{2} d v_{o}^{2}(t)$, where $\langle\cdots\rangle$ denotes an average over $f(v, t)$.

Many authors have used thermostats, of which we consider three different types [8]:

$$
\mathcal{F} f(\mathbf{v}, t)= \begin{cases}\gamma \frac{\partial}{\partial \mathbf{v}} \cdot \mathbf{v} f(\mathbf{v}, t) & \text { Gaussian } \\ -\frac{1}{2} \xi_{o}^{2}\left(\frac{\partial}{\partial \mathbf{v}}\right)^{2} f(\mathbf{v}, t) \quad \text { white noise } \\ g \frac{\partial}{\partial \mathbf{v}} \cdot \hat{\mathbf{v}} f(\mathbf{v}, t) & \text { gravity. }\end{cases}
$$

A popular thermostat, used in molecular dynamics simulations of elastic systems, is the Gaussian thermostat, which is based on Gauss' principle of least constraint $[8,16]$, and yields in the present case a friction force $\gamma \mathbf{v}$ with a negative friction coefficient. Many investigators [3,6,10,17-19] have added stochastic forces to the equations of motion in the form of Gaussian white noise. Following Ref. [8] we will also consider the gravity thermostat. The thermostat forces considered here are all scalars, but thermostat forces of a tensorial nature have also been considered, e.g., to describe uniform shear flow [20].

Montanero and Santos [8] made the interesting observation that the Boltzmann equation for the NESS distribution $f(v, \infty)$ driven by a Gaussian thermostat is identical to the equation for the scaling or similarity solution $\tilde{f}(c)$ in the freely cooling state (i.e., $\mathcal{F} f=0$ ) provided both equations are expressed in rescaled distributions. These are defined as $f(v, t)=\left[v_{o}(t)\right]^{-d} \widetilde{f}\left(v / v_{o}(t)\right)$ for the homogeneous cooling state, and as $f(v, \infty)=\left[v_{o}(\infty)\right]^{-d} \widetilde{f}\left(v / v_{o}(\infty)\right)$ for the NESS, which implies for both rescaled functions the normalizations, $\int d \mathbf{c} \widetilde{f}(c)\left\{1, c^{2}\right\}=\left\{1, \frac{1}{2} d\right\}$. 
In terms of these rescaled variables the Boltzmann equation for $\widetilde{f}(c)$ in the NESS becomes

$$
\widetilde{I}(\tilde{f})=\widetilde{\mathcal{F}} \widetilde{f}=\left\{\begin{array}{l}
\gamma \frac{\boldsymbol{\partial}}{\partial \mathbf{c}} \cdot \mathbf{c} \tilde{f}=\frac{\omega_{2}}{d} \frac{\partial}{\partial \mathbf{c}} \cdot \mathbf{c} \widetilde{f} \\
-\frac{\xi_{o}^{2}}{2 v_{0}^{2}}\left(\frac{\boldsymbol{\partial}}{\partial \mathbf{c}}\right)^{2} \tilde{f}=-\frac{\omega_{2}}{2 d}\left(\frac{\partial}{\partial \mathbf{c}}\right)^{2} \tilde{f} \\
\frac{g}{v_{o}} \frac{\boldsymbol{\partial}}{\partial \mathbf{c}} \cdot \hat{\mathbf{c}} \tilde{f}=\frac{\omega_{2}}{2\langle c\rangle} \frac{\boldsymbol{\partial}}{\partial \mathbf{c}} \cdot \hat{\mathbf{c}} \tilde{f} .
\end{array}\right.
$$

To derive the equalities in the last column, one multiplies the Boltzmann equation (4) to the left with $\int d \mathbf{c} c^{2}$, and introduces the second moment of the reduced collision operator, $\omega_{2}=-\int d \mathbf{c} c^{2} \widetilde{I}(\widetilde{f}), \quad$ to obtain $\omega_{2}=\left[-d \gamma,-d \xi_{o}^{2} / v_{o}^{2}(\infty)\right.$, $\left.-2 g\langle c\rangle / v_{o}(\infty)\right]$ respectively. These equalities are used to eliminate the parameters $\gamma, \xi_{o}^{2} / v_{o}^{2}, g / v_{o}$ in the first column. For Maxwell models, $\omega_{2}$ can be calculated exactly (see [4]) with the result $\omega_{2}=\frac{1}{4}\left(1-\alpha^{2}\right)$ for $g(\mu)=1$ and $\omega_{2}$ $=\frac{1}{2}[d /(d+1)]\left(1-\alpha^{2}\right)$ for $g(\mu)=C|\mu|$. The moment $\langle c\rangle$ $=\langle|\mathbf{c}|\rangle$ in Eq. (4) has to be calculated from the NESS distribution $\tilde{f}(c)$ in the bulk range, i.e., $c=\mathcal{O}(1)$, where $\widetilde{f}(c)$ is given in lowest approximation $[6,10]$ by the Maxwellian $\pi^{-d / 2} \exp \left(-c^{2}\right)$. This yields $\langle c\rangle=\Gamma\left(\frac{1}{2}(d+1)\right) / \Gamma\left(\frac{1}{2} d\right)$.

The Boltzmann equation (4) for the rescaled distribution function $\widetilde{f}(c)$ in the NESS not only holds for $d$-dimensional Maxwell models, but also for $d$-dimensional inelastic hard spheres, provided $\xi_{o}^{2} / v_{o}^{2}, \gamma, g / v_{o}$ are replaced by $\xi_{o}^{2} / v_{o}^{3}, \gamma, g / v_{o}^{2}$ (see discussion in Refs. [6,8]). Therefore, obtaining the solution of Eq. (4) in the NESS for all three thermostat forces above covers a large range of representative cases.

\section{HIGH ENERGY TAILS}

Following the procedure of Ref. [6] we make the ansatz of stretched exponential behavior for the high energy tail, i.e., $\widetilde{f}(c) \simeq A \exp \left(-a c^{b}\right)$ with $0<b<2$ and $a>0$, and determine $b$ and $a$ by inserting this ansatz in the rescaled Boltzmann equation (4) and requiring self-consistency. The border line case $b \rightarrow 0$ corresponds to power law tails, and $b \rightarrow 2$ corresponds to Gaussian tails.

Following Krook and $\mathrm{Wu}$ [21] and Ernst [22], we give an estimate for the collision operator $\widetilde{I}(\widetilde{f})$ for $\mathbf{c}_{1}$ being in the asymptotic range $\left(c_{1} \gg 1\right)$. If particle 1 is a fast particle, the dominant contribution to the Boltzmann collision term comes from collisions with particle 2 with velocities that are typically in the bulk range $\left[c_{2}=\mathcal{O}(1)\right]$. Consequently $\mathbf{c}_{12}$ in the collision terms (1) and (4) can be replaced by $\mathbf{c}_{1}$, i.e., $\left|\mathbf{c}_{12} \cdot \mathbf{n}\right| \simeq\left|\mathbf{c}_{1} \cdot \mathbf{n}\right|$, and the asymptotic dynamics simplifies to $\quad \mathbf{c}_{1}^{* *}=\mathbf{c}_{1}-\frac{1}{2}[1+(1 / \alpha)] \mathbf{c}_{1} \cdot \mathbf{n n} \equiv \mathbf{c}_{-} \quad$ and $\quad \mathbf{c}_{2}^{* *}=\frac{1}{2}[1$ $+(1 / \alpha)] \mathbf{c}_{1} \cdot \mathbf{n n} \equiv \mathbf{c}_{+}$. Next we make an estimate of the gain term in the collision operator under the assumption that the stretched exponential behavior holds asymptotically for large $\mathbf{c}_{1}$. To do so we consider the ratio $R\left(\mathbf{c}_{1}, \mathbf{c}_{2}\right) \equiv f_{1}^{* *} f_{2}^{* *} / f_{1} f_{2}$ for asymptotically large $\mathbf{c}_{1}$, whereas $\mathbf{c}_{2}$ is in the bulk range, i.e.,

$$
R\left(\mathbf{c}_{1}, \mathbf{c}_{2}\right) \simeq \exp \left[-a\left(c_{+}^{b}+c_{-}^{b}-c_{1}^{b}\right)\right] .
$$

The exponent is proportional to $c_{1}^{b}$ for $\alpha<1$ and $0<b<2$, except for grazing collisions, where it vanishes. This happens in a small $\theta$ interval of length $\mathcal{O}\left(1 /\left[\left(1-\alpha^{2}\right) c_{1}\right]\right)$ near $\theta$ $=\pi / 2$, where $\left(1-\alpha^{2}\right) c_{1}|\cos \theta| \simeq \mathcal{O}(1)$. Outside this interval $R$ vanishes exponentially fast, and inside $R \simeq \mathcal{O}(1)$. We insert this estimate of $R$, which is independent of $c_{2}$, in the collision terms (1) and (4), and carry out the integrations over $\mathbf{c}_{2}$ and over the angle of incidence by making the substitution $\theta=\pi / 2-x / c_{1}$. This yields the following estimate for the gain term:

$$
I_{g} \sim \int d \mathbf{n} \int d \mathbf{c}_{2}\left|\hat{\mathbf{c}}_{1} \cdot \mathbf{n}\right| s f_{1} f_{2} \sim f_{1} \delta^{s+1} / c_{1}^{s+1},
$$

where the constant $\delta \sim \mathcal{O}(1)$ and the exponent $s=\{0,1\}$ covers both collision frequencies $g(\mu)$, discussed below Eq. (1). A comparison of the gain and loss terms in Eq. (1) shows that for $\alpha<1$, the ratio $I_{g} / I_{l} \sim \mathcal{O}\left(1 / c_{1}^{s+1}\right)$ for $c_{1} \gg 1$. So, under the assumption that the stretched exponential form is valid, the gain term can be neglected in the asymptotic analysis, and the collision integral is given by $\widetilde{I}(\widetilde{f}) \simeq-\widetilde{f}\left(c_{1}\right)$. It is important to note that this estimate breaks down as $\alpha \uparrow 1$, because the $\mathbf{n}$ or $\theta$ integral converges nonuniformly in $\alpha$ near the grazing collisions, and the limit $\alpha \uparrow 1$ cannot be taken under the integral sign in the gain term.

After these preparations we insert the stretched exponential form into the Boltzmann equation (4), and match the leading exponents on both sides of the equation, as well as the coefficients in the exponents of these terms. This gives the following results for the asymptotic high energy tail of $\widetilde{f}(c) \sim \exp \left(-a c^{b}\right)$ in $d$-dimensional IMM:

$$
\begin{array}{lll}
b=0, & \text { inconsistent } & \text { (Gaussian), } \\
b=1, & a=\sqrt{2 d / \omega_{2}} & \text { (white noise), } \\
b=1, & a=2\langle c\rangle / \omega_{2} & \text { (gravity). }
\end{array}
$$

The ansatz of a stretched exponential tail for a Gaussian thermostat turns out to be inconsistent in IMM, and suggests a power law decay. The self-consistent method of [6] does not lead to a prediction. In this case, which is equivalent to the freely evolving case, has been extensively discussed in Refs. [2-4,14]. For comparison we also quote the results for $d$-dimensional inelastic hard spheres. One easily verifies that the above estimate yields for hard spheres, $\widetilde{I}_{g} \simeq$ $-c_{1} \beta_{1} \widetilde{f}\left(c_{1}\right)$ with $\beta_{1}=\int_{\mathbf{n}}\left|\hat{\mathbf{v}}_{1} \cdot \mathbf{n}\right|=\Gamma\left(\frac{1}{2} d\right) /\left[\sqrt{\pi} \Gamma\left(\frac{1}{2}(d+1)\right)\right]$ (see Ref. [4]), and one obtains immediately the results of Ref. [6], i.e., $\widetilde{f}(c) \sim \exp \left(-a c^{b}\right)$ with

$$
\begin{array}{lll}
b=1, & a=d \beta_{1} / \omega_{2} & \text { (Gaussian), } \\
b=\frac{3}{2}, & a=\sqrt{\frac{8 d \beta_{1}}{9 \omega_{2}}} \quad \text { (white noise), } \\
b=2, & \text { inconsistent } & \text { (gravity). }
\end{array}
$$




\section{CONCLUSIONS AND PERSPECTIVES}

The theoretical results for IMM in Eq. (7) and for IHS in Eq. (8) show for which cases the rescaled form of the NESS distribution function $\tilde{f}(c)$ at large energies is consistently described by stretched exponentials with $0<b<2$, where all moments exist. In IMM the case $b=0$ is a border line case, corresponding to power law tails, as discussed in Refs. $[2-4,14]$. The case of the gravity thermostat for IHS with $b=2$ is another border line case with Gaussian tails, which has been discussed in Ref. [8].

The results also show that the form of the high energy tails is by no means universal. It not only depends strongly on the type of thermostat, but also on the type of interaction model. For instance, in the case of the white noise thermostat the distributions have stretched exponential tails with $b=1$ for Maxwell models, and for IHS it is $b=3 / 2$. In the case of the Gaussian thermostat or freely cooling systems there are power law tails [2-4] in inelastic Maxwell models, and stretched exponentials with $b=1$ for inelastic hard spheres. We also observe that the tails for a given thermostat in IHS have smaller overpopulation as for the same thermostat in IMM, because the collision rate in the hard sphere case, which is proportional to $g(\mu) \sim\left|\mathbf{c}_{12}\right|$, is much more efficient in randomizing the velocities at high energies than in Maxwell models.

When comparing thermostats for a given model we note that the overpopulation decreases in the order in which the thermostats are listed. The stronger the source terms are at large velocities, the larger is the overpopulation. Weaker source terms at large velocities yield smaller overpopulations, closer to Maxwellian tails. We have not been able to determine a priori criteria for lower bounds on the source strength at large velocities, below which there always will be a Gaussian tail.

Freely cooling dissipative systems (without energy input) are totally equivalent to the special case of driving by a Gaussian thermostat, and are, therefore, included in our comprehensive analysis.

In summary, the most common scenario for spatially homogeneous driven dissipative systems is that an arbitrary initial distribution $f(v, 0)$, bounded by a Gaussian distribution at large energies - the scenario for singular initial distributions with power law tails is more complicated (see Ref. [4b])_approaches a nonequilibrium steady state (NESS) $f(v, \infty)$ with an overpopulated high energy tail. The global stability of this NESS in spatially homogeneous systems has been shown in Ref. [4b] for inelastic Maxwell models, driven by a Gaussian thermostat, or equivalently for an inelastic Maxwell model in the homogeneous cooling state without energy input.

\section{ACKNOWLEDGMENTS}

The authors acknowledge financial support from DGES (Spain) Grant No. BFM-2001 0291.
[1] G.P. Collins, Sci. Am. 284(1), 17 (2001).

[2] A. Baldassarri, U. Marini Bettolo Marconi, and A. Puglisi, (a) e-print cond-mat/0105299; (b) e-print cond-mat/0111066; (c) (private communication).

[3] E. Ben-Naim and P.L. Krapivsky, Phys. Rev. E 61, R5 (2000); P.L. Krapivsky and E. Ben-Naim, e-print cond-mat/0111044.

[4] M.H. Ernst and R. Brito, (a) e-print cond-mat/0111093; (b) e-print cond-mat/0112417.

[5] S.E. Esipov and T. Pöschel, J. Stat. Phys. 86, 1395 (1997).

[6] T.P.C. van Noije and M.H. Ernst, Granular Matter 1, 57 (1998); e-print cond-mat/9803042.

[7] J.J. Brey, M.J. Ruiz-Montero, and D. Cubero, Phys. Rev. E 54, 3664 (1996).

[8] J.M. Montanero and A. Santos, Granular Matter 2, 53 (2000); e-print cond-mat/0002323.

[9] A. Barrat, T. Biben, Z. Racz, E. Trizac, and F. van Wijland, e-print cond-mat/0110345.

[10] J.A. Carrillo, C. Cercignani, and I.M. Gamba, Phys. Rev. E 62, 7700 (2000).

[11] Th. Biben, Ph. A. Martin, and J. Piasecki, Physica A (to be published).

[12] T.P.C. van Noije, M.H. Ernst, E. Trizac, and I. Pagonabarraga, Phys. Rev. E 59, 4326 (1999).

[13] I. Goldhirsch and G. Zanetti, Phys. Rev. Lett. 70, 1619 (1993).

[14] A.V. Bobylev and C. Cercignani (unpublished).

[15] A.V. Bobylev, J.A. Carrillo, and I.M. Gamba, J. Stat. Phys. 98, 743 (2000).

[16] D. Evans and G.P. Morriss, Statistical Mechanics of Nonequilibrium Liquids (Academic Press, London, 1990).

[17] D.R.M. Williams and F.C. MacKintosh, Phys. Rev. E 54, R9 (1996).

[18] I. Pagonabarraga, E. Trizac, T.P.C. van Noije, and M.H. Ernst, e-print cond-mat/0107570.

[19] C. Bizon, M.D. Shattuck, J.B Swift, and H.L. Swinney, Phys. Rev. E 60, 4340 (1999).

[20] L. Acedo, A. Santos, and A.V. Bobylev, e-print cond-mat/0109490.

[21] T. Krook and T.T. Wu, Phys. Rev. Lett. 36, 1107 (1976).

[22] M.H. Ernst, Phys. Rep. 78, 1 (1981).

[23] B. Nienhuis (private communication). 